\title{
The identification of a genetically unique piroplasma in North American river otters (Lontra canadensis)
}

\author{
A. J. BIRKENHEUER ${ }^{1,3 *}$, C. A. HARMS ${ }^{1,2,3}$, J. NEEL ${ }^{1}$, H. S. MARR ${ }^{1}$, M. D. TUCKER ${ }^{1}$, \\ A. E. ACTON ${ }^{1,3}$, A. D. TUTTLE ${ }^{2,3}$ and M. K. STOSKOPF $\mathrm{ST}^{1,2,3}$ \\ ${ }^{1}$ North Carolina State University, College of Veterinary Medicine, 4700 Hillsborough Street, Raleigh, NC 27606, USA \\ ${ }^{2}$ North Carolina State University, Center for Marine Sciences and Technology, 303 College Circle, Morehead City, \\ NC 28557, USA \\ ${ }^{3}$ Environmental Medicine Consortium, 4700 Hillsborough Street, Raleigh, NC 27606, USA
}

(Received 22 August 2006; revised 11 October 2006; accepted 26 October 2006; first published online 11 Fanuary 2007)

\begin{abstract}
SUMMARY
During a routine health check of a wild-caught North American river otter (Lontra canadensis) small piroplasms were noted within erythrocytes. Analyses of the $18 \mathrm{~S}$ ribosomal ribonucleic acid (rRNA) gene sequences determined that this was a genetically unique organism most closely related to Babesia microti-like parasites found in other small carnivores. Subsequently 39 wild-trapped North American river otters from North Carolina were tested for the presence of piroplasma deoxyribonucleic acid (DNA) via polymerase chain reaction and piroplasma DNA was detected in $82 \%(32 / 39)$ of these samples. Sequencing of partial $18 \mathrm{~S}$ rRNA genes from selected cases determined that they were identical to the sentinel case. This report documents the existence of a genetically unique piroplasma in North American river otters and indicates that the prevalence of piroplasma in North Carolina otters is quite high. The pathogenic potential of this organism for otters or other species remains unknown.
\end{abstract}

Key words: Babesia, North American river otter, PCR, phylogenetics.

\section{INTRODUCTION}

Babesia species are tick-transmitted intraerythrocytic protozoal parasites that are in the class Piroplasmida, which includes the families Babesiidae and Theileridae (Levine, 1988). Babesia organisms were first described in cattle in 1888 and since that time over 100 Babesia species have been named. There are only a few reports of Babesia species infecting mustelids: Babesia missirolii in European badgers (Meles taxus) (Biocca and Corradetti, 1952), Babesia roubaudi in zorillas (Ictonyx striatus) (Peirce et al. 1972) and Babesia mustelae in European polecats (Mustela putorius) (Lebedeff and Tscharnotzky, 1911) respectively, and none of these has been characterized at the molecular level. To our knowledge piroplasma have not been reported or genetically characterized in otters. Characterization of the piroplasms infecting wildlife is important because it can lead to identification of zoonotic babesiosis as well as to identification of potential reservoir hosts (Penzhorn, 2006).

A number of parasitic diseases in North American river otters have been summarized in a recent review (Kimber and Kollias, 2000). Despite their

* Corresponding author: North Carolina State University, College of Veterinary Medicine, Vector Borne Disease Diagnostic Laboratory, 4700 Hillsborough Street, Raleigh, NC 27606, USA. Tel: +919 513 6193. E-mail: ajbirken@ncsu.edu aquatic nature, otters are subject to parasitism by at least 3 genera of ticks: Ixodes, Amblyomma and Dermacentor. However, tick-transmitted diseases have not been reported in North American otters and therefore these ticks were presumed to be non-pathogenic and not to act as vectors for ticktransmitted infections (Kimber and Kollias, 2000). The identification of piroplasma in North American river otters challenges this dogma. The purpose of this study was to morphologically and genetically characterize the piroplasms of North American river otters and to determine the prevalence of these piroplasms in a convenience sample of North American river otters from North Carolina.

\section{MATERIALS AND METHODS}

\section{Sources of samples}

Blood was collected via jugular venipuncture from a wild-caught North American river otter during a quarantine examination for an exhibit aquarium. Piroplasms were noted on thin, Giemsa-stained blood smears examined at $1000 \times$ magnification by light microscopy. Blood was collected aseptically from 39 North American river otters post-mortem via cardiac puncture. These otters were legally trapped in the state of North Carolina and sold to a licensed fur dealer where the sampling was performed. 
DNA isolation, amplification, cloning, sequencing and phylogenetic analyses

Total DNA was extracted from anti-coagulated whole blood or clotted blood using QIAmp blood mini kit (Qiagen, Valencia, CA) or the QIAmp tissue kit, respectively. Anti-coagulated whole blood was treated according to the manufacturer's instructions without modification. One hundred micrograms of clotted blood were processed according to the manufacturer's instructions.

Nearly full-length $18 \mathrm{~S}$ rRNA gene sequences were amplified, cloned and sequenced as previously described (Birkenheuer et al. 2003). The molecular survey was performed using a combination of 2 forward primers (5'-GCATTTAGCGATGGACCATTCAAG-3' and 5'-GCCGGCGATGTATCATTCAAG-3') and 1 reverse primer $\left(5^{\prime}\right.$ CCTGTATTGTTATTTCTTGTCACTACCTC-3') designed to amplify a 200-220 base pair amplicon from most piroplasms. The reaction conditions and cycling parameters were similar to previously described assays with minor modification (Birkenheuer et al. 2003). Equal amounts of each forward primer, $12.5 \mathrm{pmol}$, were used with each reaction.

Amplification and sequencing of a larger fragment of the 18S rRNA gene from selected samples was performed using the same combination of 2 forward primers and a different reverse primer (5'TGCTTTCGCAGTAGTTCGTC-3') that amplified an approximately 620 base-pair amplicon. The reaction conditions and cycling parameters were similar to previously described assays with minor modification. Equal amounts of each forward primer, $12.5 \mathrm{pmol}$, were used with each reaction (Birkenheuer et al. 2003). The 620 base-pair amplicons were purified with a commercial kit (QIAquick PCR purification kit, Qiagen Inc, Valencia, CA). Both purified amplicons and plasmid DNA were submitted for bi-directional sequencing on an ABI 3730 DNA sequencer (Davis Sequencing, Davis, CA, USA).

All samples were screened for the presence of PCR inhibitors by the amplification of glyceraldehyde-3-phosphate dehydrogenase pseudogene DNA as previously described (Birkenheuer et al. 2003).

The 18S rRNA gene sequence derived from this study was submitted to GenBank (see Appendix 1: bankit852522). Sequence alignments of piroplasma $18 \mathrm{~S}$ rRNA genes (Fig. 2) were constructed using Clustal $\mathrm{W}$ in the Bioedit software package (http:// www.mbio.ncsu.edu/BioEdit/bioedit.html). The final alignment consisted of 1493 nucleotide positions. Cladogram construction and bootstrap analyses were performed using parsimony methods (DNAPARS, SEQBOOT and CONSENSE; PHYLIP 3.6 package).

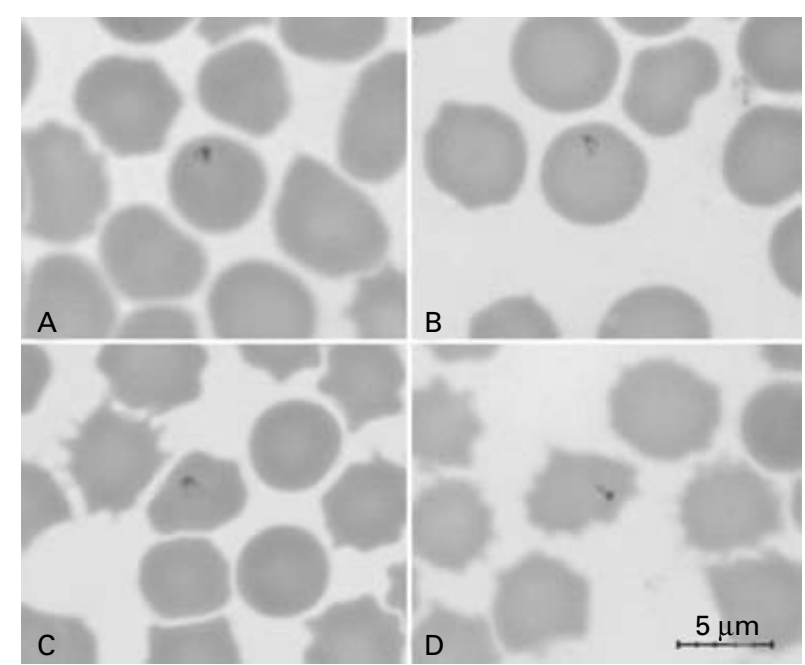

Fig. 1. Photomicrograph of merozoites identified in the blood of North American river otters. Modified WrightGiemsa stain, $1000 \times$ magnification. Round (A), oval (B), piriform (C), and matchstick (D) shapes are noted.

RESULTS

\section{Microscopical description}

Small piroplasms were identified in approximately $1 \cdot 2 \%$ of erythrocytes. These organisms were round to oval to piriform with occasional matchstick or stellate shapes noted (Fig. 1). Infected RBCs typically contained a single organism; rarely cells contained 2 . Based on measurements from 40 organisms, they varied in length from 0.75 to $2.4 \mu \mathrm{m}$ and width from 0.25 to $1.5 \mu \mathrm{m}$ with an average width and length of $0.95+/-0 \cdot 25 \mu \mathrm{m}$ by $1 \cdot 41+/-0 \cdot 43 \mu \mathrm{m}$. The organisms had pale staining cytoplasm and a thin, lightblue outer membrane. The nuclei were eccentric, associated with the outer membrane, and magenta staining, and they varied from round to oval to crescent in shape with an average diameter of $0.5 \mu \mathrm{m}$.

\section{DNA sequencing and phylogenetic analyses}

A 1733 base-pair $18 \mathrm{~S}$ rRNA gene fragment was amplified, cloned and sequenced bi-directionally from the sentinel case. The $18 \mathrm{~S}$ rRNA sequence was unique compared to the other sequences in GenBank and was determined to have the highest degree of sequence similarity $(98 \%)$ with a Babesia microti-like piroplasma isolated from dogs in northwestern Spain. Sequencing of 620 base-pair amplicons of the 18S rRNA gene including the V4 hypervariable region from 2 randomly selected cases from the convenience samples revealed $100 \%$ sequence identity with the sentinel case. Phylogenetic analyses placed the piroplasma species from the otters in a clade with $B$. microti with excellent bootstrap support $(100 \%)$ of this node (Fig. 2). Within this clade there was reasonable bootstrap support 


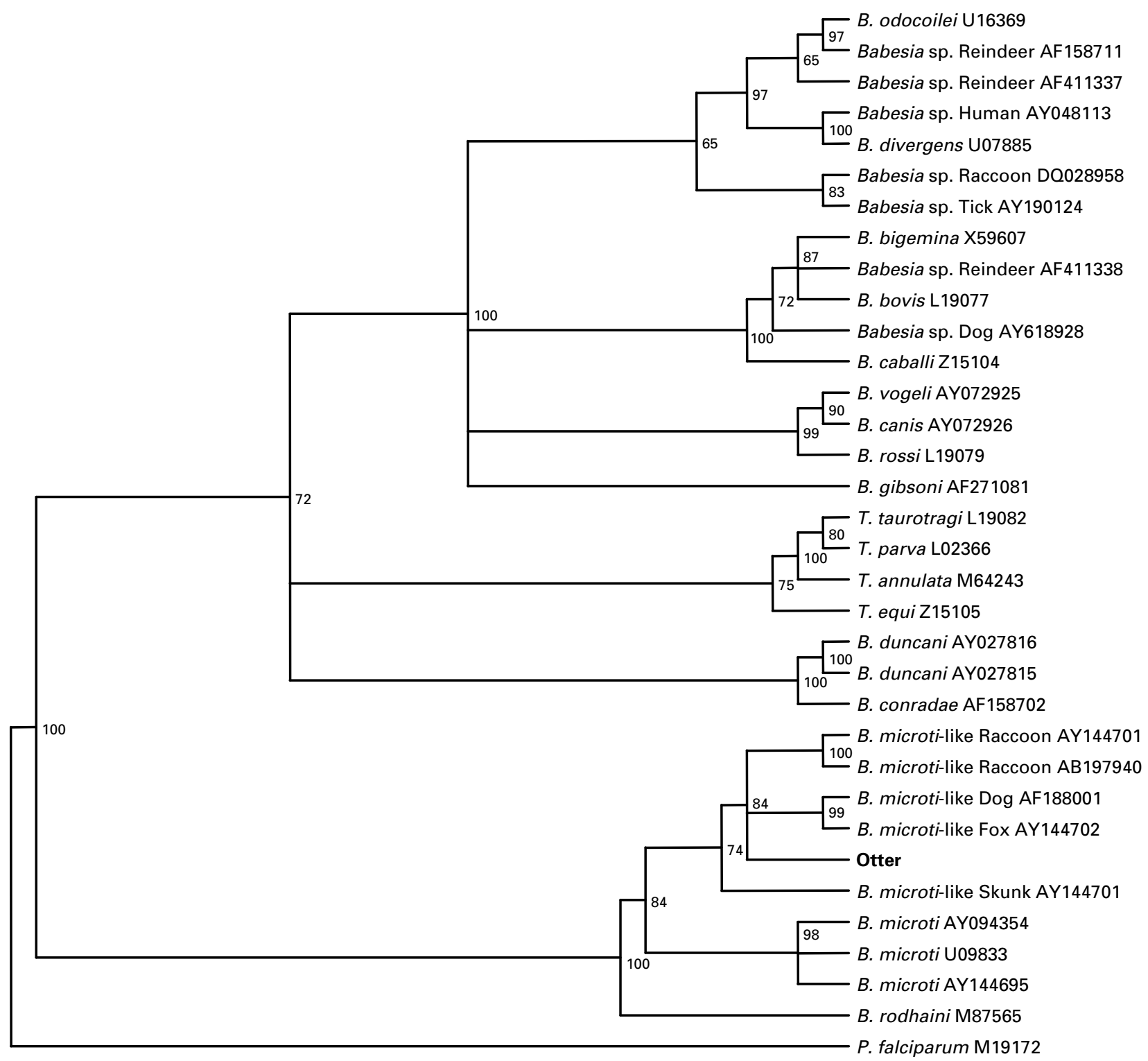

Fig. 2. The Babesia species identified in North American river otters is part of the Babesia microti-like clade. Bootstrap consensus tree showing groups that occurred in $\geqslant 60 \%$ of trees. Nodes with $<60 \%$ support have been collapsed.

Numbers by each node indicate the bootstrap value determined from 100 replicates.

$(84 \%)$ for this organism to be placed in a group with the $B$. microti-like piroplasms identified in other carnivores rather than with those identified from rodents and humans (Goethert and Telford, 2003).

\section{Molecular survey}

Babesia spp. DNA was detected by PCR in $82 \%$ $(32 / 39)$ of the samples tested based on the visualization of amplicons of the expected size after electrophoresis in an agarose gel, ethidium bromide staining and transillumination with an ultraviolet light. The presence of PCR inhibitors in all samples from which piroplasma DNA was not detected was excluded by the amplification of glyceraldehyde-3-phosphate dehydrogenase pseudogene DNA (data not shown).
If this convenience sample is representative of the otter population in North Carolina, then the prevalence of piroplasma infections is likely to be 70-90\% $(90 \% \mathrm{CI})$.

\section{DISCUSSION}

Piroplasma in North American river otters has not been documented previously. This report describes a genetically unique piroplasma identified in these otters and documents that this infection is present in a high percentage of animals. Since the primers used to perform the molecular survey are broad-range piroplasma primers and not all amplicons were sequenced it is important to note the possibility that some of these otters may actually be infected with other piroplasma species. 
The piroplasma of North American river otters appears to reside in a monophyletic clade with Babesia microti, is most closely related to the B. microti-like parasites of other small carnivores and shares the highest degree of sequence similarity with the B. microti-like organism isolated from dogs in northwest Spain. As it is unclear whether or not the organism described in this study represents a new species, subspecies or is one of the over 100 existing Babesia spp. that have not been characterized at the molecular level, the authors feel that it is inappropriate to propose it as a novel Babesia species or subspecies at this time. With the advent of molecular techniques and their application to the phylogenetics of piroplasms, the nomenclature and status of many organisms is either questionable or in a state of flux. For example, the $B$. microti-like piroplasma from dogs in northwestern Spain that appears to be closely related to the piroplasma in these North American river otters, has been referred to as 'Theileria annae.' However, there was no evidence presented to demonstrate lymphocytic stages in the life-cycle (Zahler et al. 2000). Meanwhile others, including this study, have found the piroplasma from dogs to be more closely related to $B$. microti (Goethert and Telford, 2003). In our analyses there are unresolved relationships within this class including some of the deeper branches differentiating some of the newly described 'Babesia' species (B. duncani and $B$. conradae) identified in humans and dogs, the Theileria organisms and the Babesia sensu stricto clade. To the authors' knowledge there is no comprehensive molecular phylogenetic analysis of piroplasms, and even if one existed it would be subject to change as more organisms undergo molecular characterizations and DNA sequence analysis. The 'final' taxonomic resting place of the piroplasma of otters characterized in this study, especially in the shallow branches of the tree, cannot be determined with the currently available data, However, the authors feel there is strong support for this organism to be placed in the B. microti-like clade of organisms.

The pathogenic potential of the parasite described in this manuscript for otters or other species has not been investigated. The index case was in good body condition, exhibited no haematological or serum biochemical abnormalities (Tocidlowski et al. 2000), and continues to do well in captivity without treatment. Further studies are indicated including association of babesiosis with clinical disease, epidemiology of the infection in North Carolina and other areas in which the North American river otter is endemic, screening other otter species for babesiosis, and the identification of competent tick vectors and the life-cycle of the organism. The characterization of Babesia spp. of wild mammals may help in the recognition of potential zoonotic babesiosis.

The authors would like to acknowledge Stuart E. May, Director of Husbandry and Operations at the NC Aquarium at Pine Knoll Shores, and Antech Diagnostics for their assistance.

\section{REFERENCES}

Biocca, E. and Corradetti, A. (1952). Babesia missirolii n. sp., a badger (Meles taxus) parasite. Annali dell'Istituto superiore di sanità 15, 312-314.

Birkenheuer, A. J., Levy, M. G. and Breitschwerdt, E. B. (2003). Development and evaluation of a seminested PCR for detection and differentiation of Babesia gibsoni (Asian genotype) and B. canis DNA in canine blood samples. Fournal of Clinical Microbiology 41, 4172-4177.

Goethert, H. K. and Telford, S. R. 3rd (2003). What is Babesia microti? Parasitology 127, 301-309.

Kimber, K. R. and Kollias, G. V. 2nd (2000). Infectious and parasitic diseases and contaminant-related problems of North American river otters (Lontra canadensis): a review. Fournal of Zoo and Wildlife Medicine 31, 452-472.

Lebedeff, W. and Tscharnotzky, A. (1911). Ein neuer Parasit im Blute des Iltis, Microsoma mustelae. Zentralblatt für Bakteriologie, Parasitenkunde, Infektionskrankheiten und Hygiene. Zweite naturwissenschaftliche Abt.: Allgemeine, landwirtschaftliche und technische Mikrobiologie 58, 625-631.

Levine, N. D. (1988). The Protozoan Phylum Apicomplexa. CRC Press, Boca Raton, Fl, USA.

Levy, M. G. and Ristic, M. (1980). Babesia bovis: continuous cultivation in a microaerophilous stationary phase culture. Science 207, 1218-1220.

Peirce, M. A., Bray, R. S., Ashford, R. W. and Garnham, P. C. C. (1972). A piroplasm of the zorilla (Ictonyx striatus) [Babesia roubaudi-Ed.]. Bulletin de la Société de pathologie exotique et de ses filiales $\mathbf{6 5}$, 663-668.

Penzhorn, B. L. (2006). Babesiosis of wild carnivores and ungulates. Veterinary Parasitology 138, 11-21.

Tocidlowski, M. E., Spelman, L. H., Sumner, P. W. and Stoskopf, M. K. (2000). Hematology and serum biochemistry parameters of North American river otters (Lontra canadensis). Fournal of Zoo and Wildlife Medicine 31, 484-490.

Zahler, M., Rinder, H., Schein, E. and Gothe, R. (2000). Detection of a new pathogenic Babesia microti-like species in dogs. Veterinary Parasitology 89, 241-248. 
APPENDIX 1

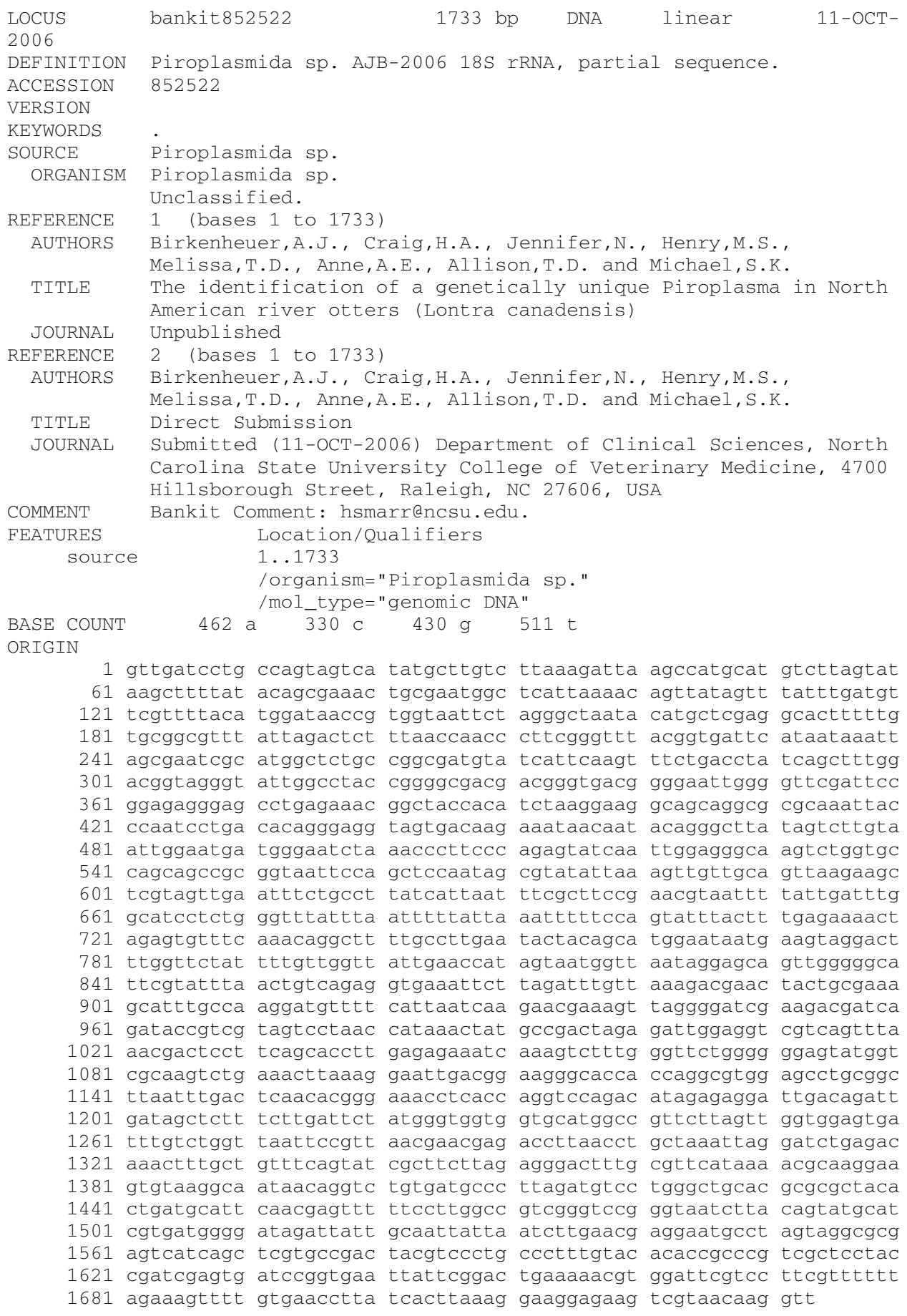

